\title{
La ecuación de la recta en la ergonomía
}

DOI: $10.46932 / \mathrm{sfjdv2n1-065}$

Received in: November 1st, 2020

Accepted in: December 30th, 2020

\section{María de Lourdes Olivares Estrada}

Dr. en Administración y Políticas Públicas.

Centro de Estudios Tecnológicos Industrial y de Servicios No. 64, Toluca, México.

Full address: Av. Benito Juárez Esq. Juan Escutia S/N, Col. San Mateo Oxtotitlán, Toluca Edo. Méx., México. C.P. 50100

E-mail:mloe09@yahoo.com.mx

\section{Omar García de la Rosa}

Dr. en Administración y Políticas Públicas.

Instituto Tecnológico de Toluca, Toluca México.

Full address: Av. Tecnológico S/N, col. Agrícola Bellavista, Metepec Edo. Méx., México. C.P. 52149

E-mail: omargarcia_1978@hotmail.com

\section{Héctor García de la Rosa}

Dr. en Administración y Políticas Públicas.

Instituto Tecnológico de Toluca, Toluca México.

Full address: Av. Tecnológico S/N, col. Agrícola Bellavista, Metepec Edo. Méx., México. C.P. 52149

E-mail: hegar71@yahoo.com.mx

\section{Ceciliano Hernández Leonardo}

Maestría en Ciencias (Física) .

Instituto Tecnológico de Toluca, Toluca México.

Full address: Av. Tecnológico S/N, col. Agrícola Bellavista, Metepec Edo. Méx., México. C.P. 52149

E-mail: lcecilianoh@yahoo.com.mx

\section{Francisco García Ramirez}

Dr. en Derecho.

Centro de Estudios en Ciencias Jurídicas y Criminológicas, Toluca Méx.

Full address: Av. Baja velocidad (Paseo Tollocan) No. 519, Metepec Edo. Méx., México. C.P. 52175

E-mail: elydedy@hotmail.com

\section{RESUMEN}

Se presenta un estudio basado en un análisis ergonómico, para proponer una adaptación de las condiciones de operación con la innovación de prácticas del sistema productivo. utilizando para ello las metodologías sugeridas en la Guía Técnica Española, OWAS y RULA. Se realizó la evaluación por los métodos propuestos, contrastando sus resultados con un modelo virtual generado en Autocad y un modelo analítico resultado del análisis de variables de la guía técnica española. Esto es un hallazgo importante dado que con una ecuación de la recta obtenida a partir de un modelo de regresión lineal, permite determinar el porcentaje de protección o el nivel de daño de un trabajador sometido a cargas sobre el aparato musculo esquelético, de los cuales se encontró un nivel de riesgo elevado para el trabajador en el desempeño de sus tareas. Por consiguiente se pensó en alternativas sustentables para abatir tal problemática.

Palabras Clave: ecuación de la recta, ergonomía, problemas musculo esquelético, protección a la salud. 


\section{INTRODUCCIÓN}

Esta investigación sobre trabajo agrícola, aplicó la ergonomía como lo recomendado por Chiner, Mas y Alcaine [1], mediante un estudio de campo con la comparación de tres metodologías complementarias que cuantificaron la exposición del trabajador a cargas excesivas y movimientos repetitivos como se describe gráficamente en la siguiente figura:

Fig. 1. Aplicación de Metodologías a esta investigación

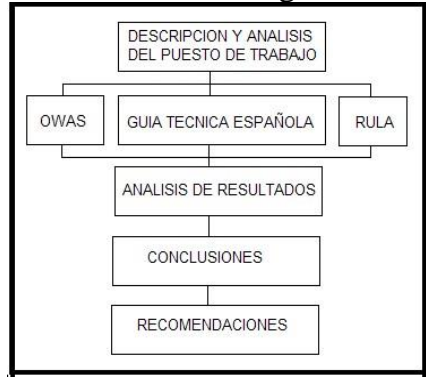

En el área de producción agrícola, el cultivo de sorgo tiene relevancia a nivel mundial como hace referencia Báez [2] y México ocupa entre el cuarto y quinto lugar productivo. Dada la importancia del tema y al indagar sobre la producción de sorgo a nivel nacional encontramos de acuerdo con INEGI [3] que existen regiones donde la cosecha se realiza aún manualmente y en algunas parcelas o ejidos se presentan los trabajadores sin equipo de protección personal o no lo quieren utilizar porque les estorba justificando incomodidad.

La tabla I muestra a los productores de sorgo en una creciente producción en los últimos años

Tabla I

PRODUCCIÓN DE SORGO

\begin{tabular}{|c|c|c|c|}
\hline \multicolumn{4}{|c|}{ Producción nacional en ciclo primavera verano en 2012} \\
\hline Estado & Supenicis(Has) & Producción(Ton) & Minicipios productores \\
\hline Guanajuab & 252,181 & $1,287,578$ & $\begin{array}{l}\text { Calaya, Abasolo, acam bero, Cortazar, } \\
\text { Ceramano, Iapuata, Penjamo, Salananca, } \\
\text { Salvatiera, Valle de Santiago, Villagran, } \\
\text { Yurria yManvelDoblado }\end{array}$ \\
\hline Michoeran & 117,234 & 436,192 & $\begin{array}{l}\text { Apatzingan Tepalcatepec, Penjazillo, La } \\
\text { pieded, Anganacutio, Jose Sia to Verduzco } \\
\text { y Alvaro Oregón }\end{array}$ \\
\hline Tamaulipas & 234,272 & 205,742 & $\begin{array}{l}\text { Abasdo, Soto la Marina, Altzn ira, } \\
\text { Gonzalez, Nante, Casas, Giemes, llera, } \\
\text { Padilla, San Carlos }\end{array}$ \\
\hline Morelos & 41,375 & 188899 & $\begin{array}{l}\text { Arochizpan, A yala, Cuevtla, Jantelako, } \\
\text { Jonacalepe, Puente de I tla, Ten oac, } \\
\text { Yecapin ta y Zacatepes }\end{array}$ \\
\hline Puebla & 20,993 & 53,223 & 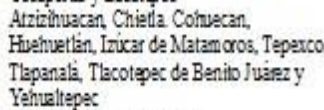 \\
\hline San Luis Potosi & 28984 & 39,983 & Bosno Tamuin y Cerritos \\
\hline Oraca & 16,680 & 38086 & $\begin{array}{l}\text { Jvchiten de Zaragora, Reforma de Pineda, } \\
\text { San Francisco del Mar, San Francisco } \\
\text { Irhuatan, San Pedro Tapanateper, Santiago } \\
\text { Niltepec, Santo Doen ingo y Uhión Hidalgo }\end{array}$ \\
\hline
\end{tabular}

La tarea a desarrollar es el corte de la panoja o mazorca (implica movimientos alternativos repetitivos) y su recolección es en ayates o en tambos (carga de hasta $30 \mathrm{Kg}$. en los hombros o manos), 
desde la surcada hasta una camioneta o un remolque. Debido a la magnitud de la carga, y a las posturas que el trabajador tiene que adoptar, es posible que el trabajador sufra daños a su salud como trastornos musculo-esqueléticos trayendo consecuencias tanto para el trabajador como para la empresa en forma económica y en productividad, de la misma manera, evitar que el trabajador no sufra daños corporales durante el desempeño de su tarea o se minimicen, y por ende las pérdidas económicas para el trabajador por concepto de pagos de servicios de salud disminuyan, con esto los trabajadores y sus familias elevará su calidad de vida.

\section{DESARROLLO}

La recolección de la cosecha de sorgo en algunas regiones se lleva a cabo utilizando maquinaria y equipo especializado mientras que en otras, por condiciones económicas, geográficas y culturales, solo es posible el corte de la planta en forma manual como se aprecia en la figura2.

Fig. 2. Cosecha manual de sorgo

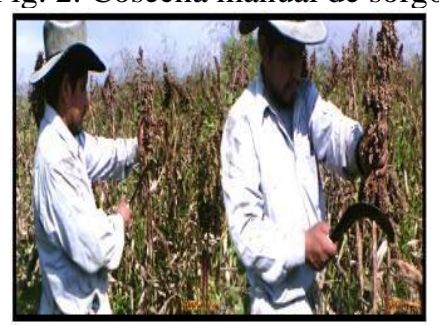

En esta actividad se usa una hoz o un machete y se va acumulando en montones sobre la parcela o se transporta en camionetas al lugar de la molienda, según se aprecia en la figura 3.

Fig. 3. Cosecha y transporte de sorgo.

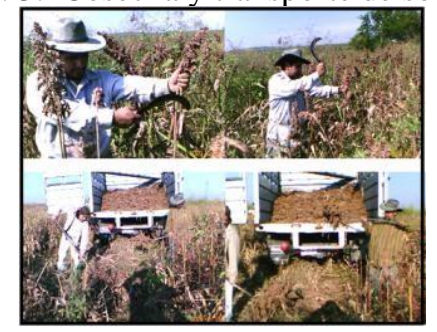

En este puesto de trabajo se tienen cargas entre 30 a $40 \mathrm{Kg}$ de peso con altas frecuencias de corte y se llega a caminar hasta unos 30 metros sobre la surcada para descargar el producto. Los resultados para la posición 1 y 2, se consideran sometiendo al trabajador a cargas de $25 \mathrm{Kg}$ recomendados y $40 \mathrm{Kg}$ para trabajadores jóvenes y entrenados mientras que en la posición 3. Se consideraron $13 \mathrm{Kg}$ recomendados y 20.8 para trabajadores jóvenes y entrenados. De acuerdo a la metodología se llenaron los siguientes formatos:

La tabla II presenta los resultados obtenidos con la aplicación de la Guía Técnica Española de 
acuerdo con el Instituto Nacional de Seguridad e Higiene en el Trabajo [4] sobre 3 posturas promedio definidas sobre una población de ocho trabajadores, donde se verifica el peso teórico o recomendado contra el peso real o carga máxima a que se somete al trabajador, el desplazamiento vertical o levantamiento de la carga, en conjunto con diferentes factores dados por el algoritmo del método y donde finalmente la guía técnica otorga una recomendación del manejo de la carga real a que debería someterse al trabajador para reducir el nivel de riesgo a su salud por daños musculo - esqueléticos.

Tabla II

Resultados de la guía técnica española

\begin{tabular}{|l|r|r|r|}
\hline & \multicolumn{3}{|c|}{ Calculo de peso aceptable } \\
\hline Posicion & Peso Teorico (Kg) & Desplazamienta vertical \\
\hline 1 Recomendado & 25 & 0.87 \\
\hline 1 Maximo & 40 & 0.87 \\
\hline 2 Recomendado & 25 & 0.87 \\
\hline 2 Maximo & \multicolumn{3}{|r|}{0.87} \\
\hline 3 Recomendado & 40 & 0.87 \\
\hline 3 Maximo & 13 & 0.87 \\
\hline Factor Giro & Factor Agarre & Factor Frecuencia & Peso Recomendado(Kg) \\
\hline 0.9 & 1 & 0.85 & 16.63 \\
\hline 0.9 & 1 & 0.85 & 26.62 \\
\hline 0.8 & 1 & 0.85 & 14.79 \\
\hline 0.8 & 1 & 0.85 & 23.66 \\
\hline 0.8 & 1 & 0.85 & 7.69 \\
\hline 0.8 & 1 & 0.85 & 23.66 \\
\hline
\end{tabular}

En las Tablas III,IV y V se presentan los resultados de las mediciones para el Método OWAS, el cual se realiza a partir de un análisis cualitativo como el mostrado en las figuras 4,5 y 6 , en el que se consideraron 3 posiciones del trabajador de corte de sorgo y comparadas contra las imágenes establecidas por la metodología.

Fig. 4 Posición 1 para OWAS

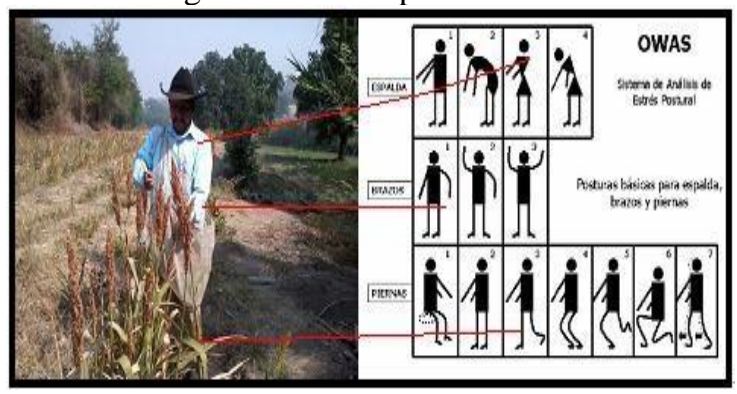


Tabla III

Resultados OWAS Para Posición 1

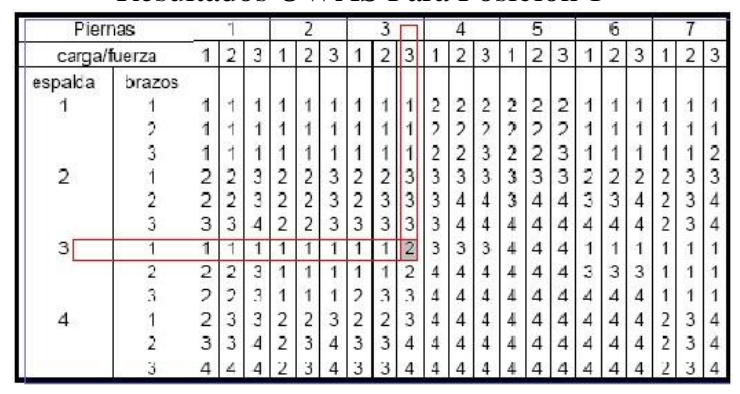

Fig. 5 Posición 2 para OWAS

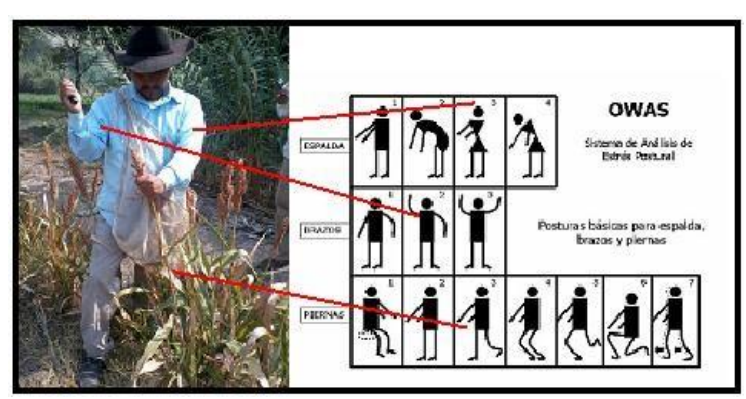

Tabla IV

Resultados OWAS para posición 2

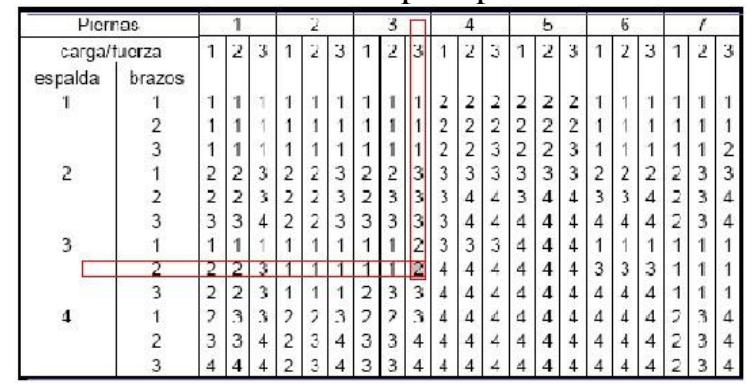

Fig. 6 Posición 3 OWAS

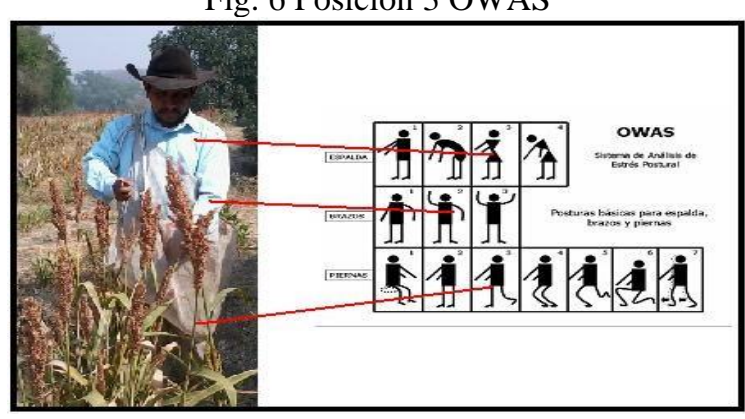


Tabla V

Resultados OWAS para posición 3

\begin{tabular}{|c|c|c|c|c|c|c|c|c|c|c|c|c|c|c|c|c|c|c|c|c|}
\hline \multicolumn{2}{|c|}{ Piernas } & \multicolumn{3}{|c|}{1} & \multicolumn{2}{|c|}{2} & \multicolumn{3}{|c|}{3} & \multicolumn{3}{|c|}{1} & \multicolumn{3}{|c|}{5} & \multicolumn{2}{|r|}{6} & \multicolumn{3}{|c|}{7} \\
\hline cargal & terza & 1 & & 3 & 1 & 5 & 31 & 2 & 3 & $\mid 1$ & 2 & 3 & 1 & 2 & 3 & 1 & 23 & & & 3 \\
\hline espada & brazos & & & & & & & & & & & & & & & & & & & \\
\hline 1 & 1 & & 1 & & & & & & & 2 & 2 & 2 & 2 & 2 & & 1 & $\begin{array}{ll}1 & 1\end{array}$ & & & 1 \\
\hline & 2 & 1 & 1 & 1 & 1 & & 1 & 1 & 1 & $\mid 2$ & 2 & 2 & 2 & 2 & 2 & 1 & 11 & 1 & 1 & 1 \\
\hline & 3 & 1 & 1 & 1 & 1 & & 1 & & 1 & 2 & 2 & 3 & 2 & 2 & 3 & 1 & 11 & 1 & 1 & 2 \\
\hline 2 & 1 & 2 & 2 & 3 & 2 & & 2 & $t$ & 3 & 3 & 3 & 3 & 3 & 3 & 3 & 2 & 22 & 2 & 3 & 3 \\
\hline & 2 & 2 & 2 & 3 & & & & & 3 & 3 & 4 & 4 & 3 & 4 & 4 & 3 & 34 & 2 & 3 & 4 \\
\hline & 3 & 3 & 3 & 1 & 2 & & 3 & 3 & 3 & 3 & 1 & 1 & 1 & 4 & 1 & 1 & 11 & 2 & 3 & 1 \\
\hline 3 & 1 & 1 & 1 & 1 & 1 & & 1 & & 2 & 3 & 3 & 3 & 4 & 4 & 4 & 1 & 11 & 1 & 1 & 1 \\
\hline & 2 & 2 & 2 & 3 & 1. & 1 & 1. & & 2 & 4 & 4 & 4 & 4 & 4 & 4 & 3 & 33 & 1 & 1 & 1 \\
\hline & 3 & 2 & 2 & 3 & 1. & & & & 3 & 4 & 4 & 4 & 4 & 4 & 4 & 4 & 44 & 1 & 1 & 1 \\
\hline 4 & 1 & 2 & 3 & 3 & 22 & & & & 3 & 4 & 4 & 4 & 4 & 4 & 4 & 4 & 44 & 2 & 3 & 4 \\
\hline & 2 & 3 & & 4 & 2 & & & & 4 & 4 & 4 & 4 & 4 & 4 & 4 & 4 & $\begin{array}{ll}44 \\
\end{array}$ & 2 & 3 & 4 \\
\hline & 3 & & & & & & & & & & & & 4 & 4 & & 4 & $\begin{array}{ll}44 \\
\end{array}$ & & & \\
\hline
\end{tabular}

Posteriormente se realizó la evaluación con el Método Rula el cual separa al cuerpo humano en miembros superiores e inferiores y se consideraron al igual que en los otros métodos tres posiciones promedio de mediciones sobre 8 personas, como se aprecia en las figuras 7,8 y 9 :

Fig. 7 Resultados Rula para Posición 1,

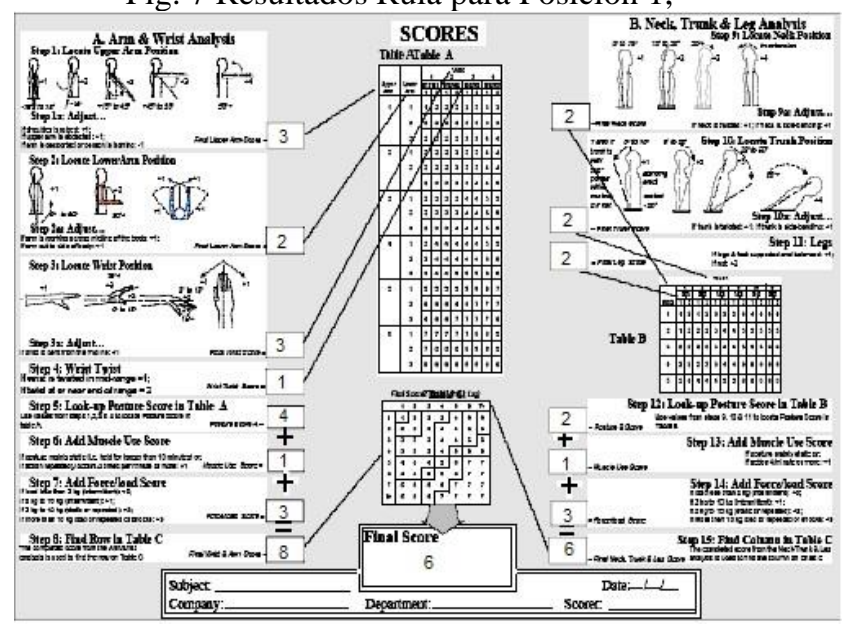

Fig. 8 Resultados Rula para Posición 2.

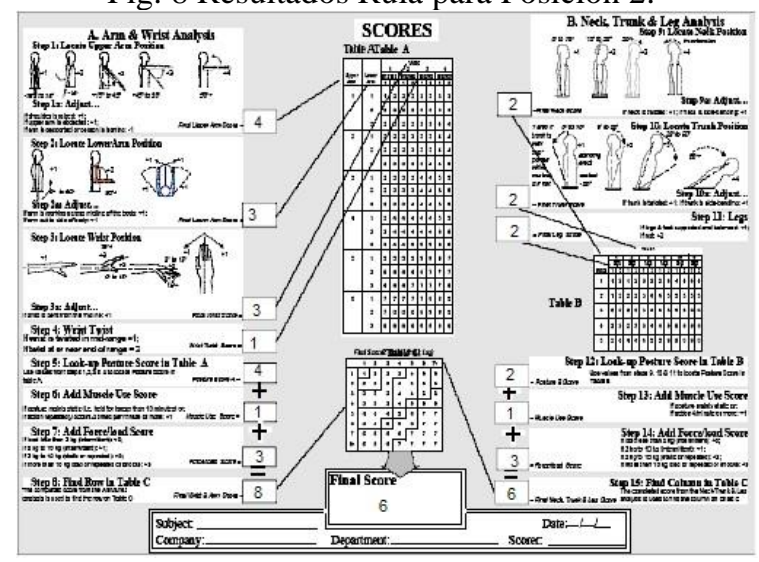


Fig. 9. Resultados RULA para posición 3

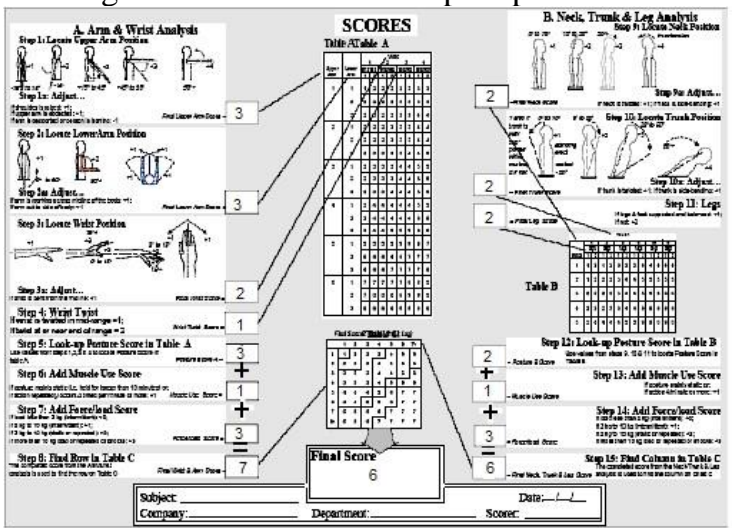

Posteriormente se sometió la misma fotografía a la medición con el software Autocad y una ampliación de la imagen para visualizar las mediciones como se ilustra en la figura 10:

Fig 10. Análisis de imagen con Autocad

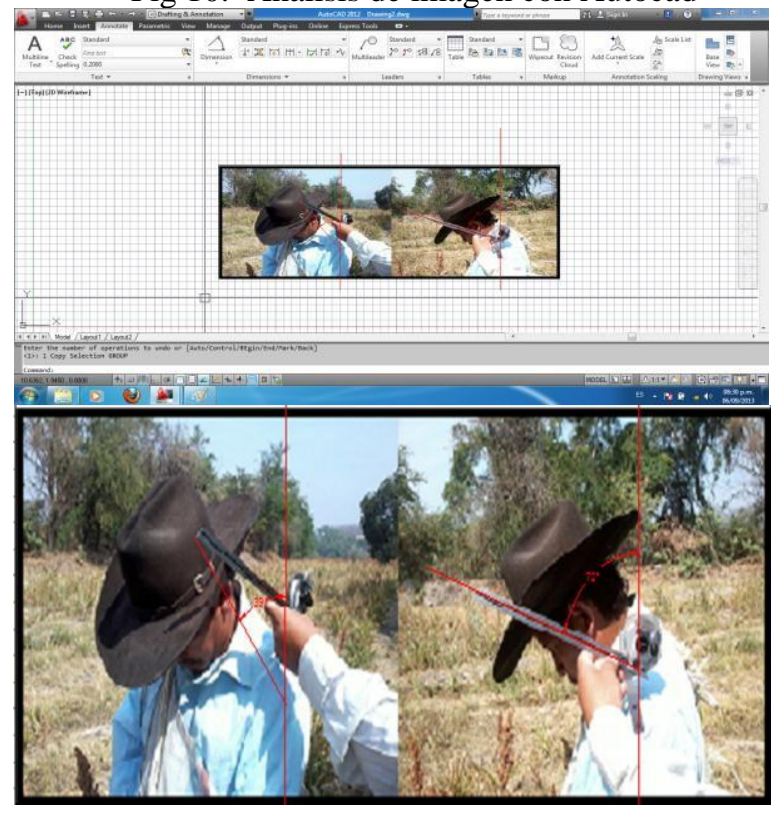

Y se observa que en el primer caso es de 39 grados y en el segundo caso es de 72 grados.

A continuación se presentan resultados derivados de la aplicación de un método gráfico a partir de las recomendaciones de la guía técnica española, en donde se encontró un polinomio de ajuste mediante extrapolación y ajuste de tendencia central que generaron una ecuación lineal de primer grado que sirve como un nuevo método ergonómico, en la figura 11 se presenta la ecuación de la recta obtenida para realizar análisis ergonómico: 
Fig. 11. Ajuste de curva para Guía técnica

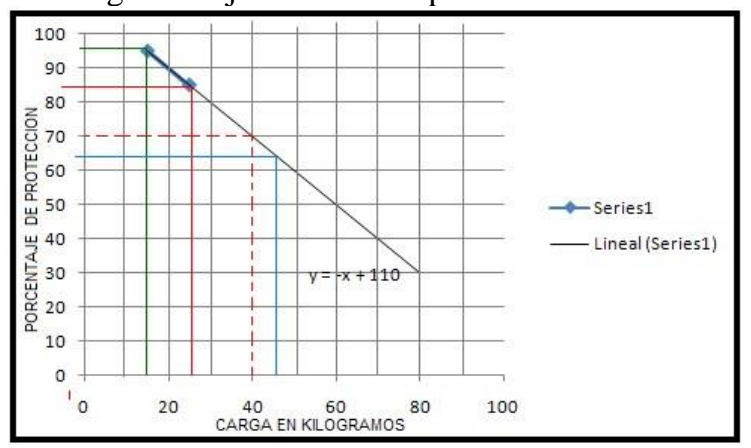

La ecuación de la forma:

$$
Y=-X+110
$$

Nos permite predecir que bajo el comportamiento de carga expresada en Kilogramos y que la relacionamos con la variable $\mathrm{X}$, se obtiene un porcentaje de protección del trabajador y la asociamos como la variable dependiente Y. Esto nos permite comprender que el alcance de las matemáticas puede extenderse hacia otras disciplinas de estudio y en este caso permite simplificar el estudio y aportar al campo de la ergonomía una herramienta tanto gráfica como analítica para predecir el nivel de protección a los trabajadores expuestos a cargas manuales y repetitivas con la finalidad de proteger la salud de las personas involucradas en las tareas.

\section{CONCLUSIONES}

El caso del análisis ergonómico de los cortadores de sorgo presentado es representativo para diversas regiones de México, el aporte más importante que puede realizar esta investigación es despertar el interés de académicos, empresarios y políticos por la aplicación de la ergonomía a la mejora y/o protección de la salud, seguridad de los trabajadores mexicanos. Con la aplicación de las metodologías seleccionadas se observó que: Los resultados para la posición 1 y 2, se consideran sometiendo a los trabajadores a cargas de $25 \mathrm{Kg}$ recomendados y $40 \mathrm{Kg}$ para trabajadores jóvenes y entrenados mientras que en la posición 3, se consideraron $13 \mathrm{Kg}$ recomendados y 20.8 para trabajadores jóvenes y entrenados, aunque realmente se transportan cargas que oscilan entre $30-40 \mathrm{Kg}$ para trabajadores en general, por lo que esta ficha clasifica el riesgo como "Riesgo no tolerable" y se requiere la reducción del riesgo. Para cada una de las tres posiciones analizadas con el método OWAS se obtuvo una puntuación final de dos y representa una postura con posibilidad de causar daño al sistema músculo-esquelético. También el método RULA con los valores de seis para cada una de las tres posiciones, plantea un rediseño de la tarea, se presume que el desarrollo de esas actividades puede desarrollar problemas de salud debidas a la ocupación.

Un hecho importante a lo largo de esta investigación es el hallazgo de una ecuación de la recta que puede ser utilizada para determinar si la cargas a las que son sometidos los trabajadores pueden causar algún nivel de daño a su sistema musculo-esquelético o el trabajador se encuentra en un nivel o rango de protección 
adecuada. Este hallazgo representa un aporte de la comunidad Mexicana al campo de la ergonomía lo que implica que las Matemáticas se encuentran inmersas en las diferentes ciencias y en cualquier tiempo.

De acuerdo a lo anterior se debe organizar el trabajo de forma que exista variación de tareas, ciclos de trabajo más largos, mecanización, mayor autonomía y control por parte del trabajador, introducción de pausas y, en definitiva, equilibrio entre las exigencias del trabajo y la capacidad del trabajador.

Debido a estas recomendaciones, se optó por mecanizar la actividad y en Rancho el Bufadero se construyó un equipo de corte mecanizado accionado por un tractor agrícola, el cual realiza la actividad (Figura12). El estudio de ergonomía aplicado, sirvió para motivar a esta organización de producción agrícola a desarrollar un equipo de trabajo diferente y apoyar su eslogan que dice: "Por un México

Autosuficiente"

Fig. 12. Implementación de Sistema mecanizado de corte de sorgo

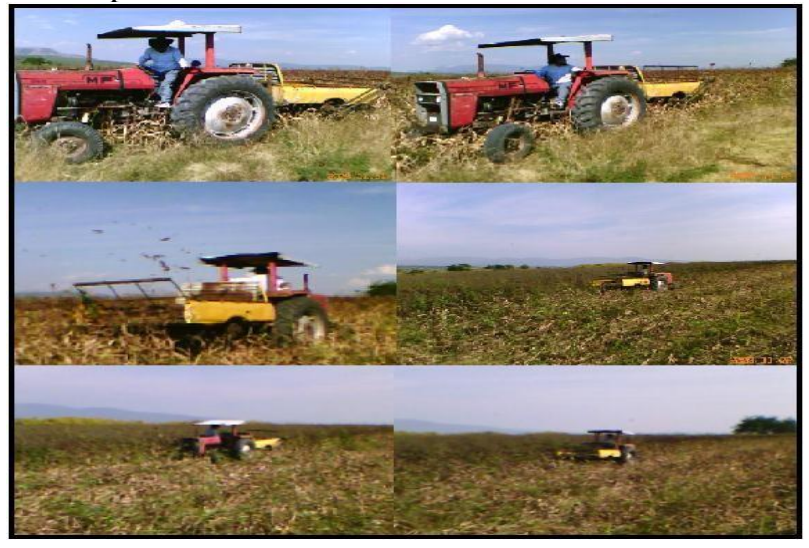




\section{REFERENCIAS}

[1]Mercedes Chiner Dasi, J. Antonio Diego Mas, Jorge Alcaide Marzal. (2011).Laboratorio de Ergonomía. Valencia, España: Alfa-omega.

[2]Báez Rodríguez Juan (2012), Producción y expectativas del sorgo en México. D.F., México: SAGARPA.

[3]INEGI Censo agropecuario en México (2007).

[4]Instituto Nacional de Seguridad e Higiene en el Trabajo INSHT (2003), Guía Técnica para la evaluación y prevención de los riesgos relativos a la manipulación manual de cargas. Barcelona, España: Ministerio del Trabajo.

[5]Mondelo R Pedro, Gregori Enrique, Blasco Joan, Barrau Pedro (2007).Ergonomía 3, Diseño de Puestos de Trabajo. Barcelona, España: Alfa-Omega [6]OIT (2004), La prevención de los accidentes, Ginebra, Suiza: Alfa-Omega. 